\title{
An Elementary Proof of the Restricted Invertibility Theorem*
}

\author{
Daniel A. Spielman \\ Department of Computer Science \\ Program in Applied Mathematics \\ Yale University
}

\author{
Nikhil Srivastava \\ Department of Computer Science \\ Yale University
}

May 28, 2018

\begin{abstract}
We give an elementary proof of a generalization of Bourgain and Tzafriri's Restricted Invertibility Theorem, which says roughly that any matrix with columns of unit length and bounded operator norm has a large coordinate subspace on which it is well-invertible. Our proof gives the tightest known form of this result, is constructive, and provides a deterministic polynomial time algorithm for finding the desired subspace.
\end{abstract}

\section{Introduction}

In this note we study the following well-known theorem of Bourgain and Tzafriri.

Theorem 1 (Restricted Invertibility [3]). There are universal constants $c, d>0$, such that whenever $L$ is a linear operator on $\ell_{2}^{n}$ with $\left\|L e_{i}\right\|=1$ for the canonical basis vectors $\left\{e_{i}\right\}_{i \leq n}$, one can find a subset $\sigma \subset[n]$ of cardinality

$$
|\sigma| \geq c n /\|L\|_{2}^{2}
$$

for which

$$
\left\|\sum_{i \in \sigma} a_{i} L e_{i}\right\|^{2} \geq d \sum_{i \in \sigma}\left|a_{i}\right|^{2}
$$

for all scalars $\left\{a_{i}\right\}_{i \in \sigma}$.

This theorem has had significant applications in the local theory of Banach spaces and in the study of convex bodies in high dimensions. It is also considered a step towards the resolution of the famous Kadison-Singer conjecture, which asks if there exists a partition of $[n]$ into a constant number of subsets $\sigma_{1}, \ldots, \sigma_{k}$ for which (1) holds. Recently, the theorem has attracted attention in numerical analysis due to its connection with the column subset selection problem, which

\footnotetext{
${ }^{*}$ This material is based upon work supported by the National Science Foundation under grants CCF-0634904, CCF-0634957 and CCF-0915487. Any opinions, findings, and conclusions or recommendations expressed in this material are those of the author(s) and do not necessarily reflect the views of the National Science Foundation.
} 
seeks to select a 'representative' subset of columns from a given matrix. In particular, Tropp [6] has developed a randomized polynomial time algorithm which finds the subset $\sigma$ efficiently.

Bourgain and Tzafriri's proof of Theorem 11 uses probabilistic and functional analytic techniques and is non-constructive. In the original paper the theorem was shown to hold for $c=d \sim \frac{1}{10^{72}}$. Later on [4], the same authors proved it for $c=c(\epsilon)=c^{\prime} \epsilon^{2}$ and $d=(1+\epsilon)^{-1}$ for every $0<\epsilon<1$, where $c^{\prime}$ is a universal (tiny) constant. They were interested in the case when $\epsilon$ is small; the quadratic dependence of $c(\epsilon)$ on $\epsilon$ was shown to be necessary in [2]. In another regime, modern methods can be used to obtain the constants $c=1 / 128$ and $d=1 / 8 \sqrt{2 \pi}[5,6]$.

In this note, we present a short proof that uses only basic linear algebra, achieves much better constants, and contains a deterministic $O\left(n^{4}\right)$ time algorithm for finding the set $\sigma$. Our method of proof involves building $\sigma$ iteratively using a 'barrier' potential function. Such a method was used by Batson and the authors in [1] to construct linear size spectral sparsifiers of graphs.

Specifically, we prove the following generalization of Theorem 1, in which $\|\cdot\|_{2}$ refers to the spectral (i.e., operator) norm and $\|\cdot\|_{F}$ refers to the Frobenius (i.e., Hilbert-Schmidt) norm.

Theorem 2. Suppose $v_{1}, \ldots v_{m} \in \mathbb{R}^{n}, \sum_{i} v_{i} v_{i}^{T}=I$, and $0<\epsilon<1$. Let $L: \ell_{2}^{n} \rightarrow \ell_{2}^{n}$ be a linear operator. Then there is a subset $\sigma \subset[m]$ of size $|\sigma| \geq\left\lfloor\epsilon^{2} \frac{\|L\|_{F}^{2}}{\|L\|_{2}^{2}}\right\rfloor$ for which $\left\{L v_{i}\right\}_{i \in \sigma}$ is linearly independent and

$$
\lambda_{\min }\left(\sum_{i \in \sigma}\left(L v_{i}\right)\left(L v_{i}\right)^{T}\right)>\frac{(1-\epsilon)^{2}\|L\|_{F}^{2}}{m},
$$

where $\lambda_{\min }$ is computed on $\operatorname{span}\left\{L v_{i}\right\}_{i \in \sigma}$.

This form of generalization was introduced by Vershynin [7] in his study of contact points of convex bodies via John's decompositions of the identity. It says that given any such decomposition and any $L: \ell_{2}^{n} \rightarrow \ell_{2}^{n}$, there is a part of the decomposition on which $L$ is well-invertible whose size is proportional to the stable rank $\frac{\|L\|_{F}^{2}}{\|L\|_{2}^{2}}$.

The original form of Bourgain and Tzafriri's theorem follows quickly from Theorem 2 with constants

$$
c(\epsilon)=\epsilon^{2} \quad \text { and } \quad d(\epsilon)=(1-\epsilon)^{2}
$$

by taking $\left\{v_{i}\right\}$ from the standard basis $\left\{e_{i}\right\}_{i \leq n}$ and assuming $\left\|L e_{i}\right\|=1$. This dominates previous bounds in all regimes, for $\epsilon$ small and large.

\section{Proof of the Theorem}

We will build the matrix $A=\sum_{i \in \sigma}\left(L v_{i}\right)\left(L v_{i}\right)^{T}$ by an iterative process that adds one vector to $\sigma$ in each step. The process will be guided by the potential function 1

$$
\begin{aligned}
\Phi_{b}(A) & =\sum_{i}\left(L v_{i}\right)^{T}(A-b I)^{-1}\left(L v_{i}\right) \\
& =\operatorname{Tr}\left[L^{T}(A-b I)^{-1} L\right] \quad \text { since } \sum_{i} v_{i} v_{i}^{T}=I,
\end{aligned}
$$

\footnotetext{
${ }^{1}$ This potential function was inspired by Stieltjes transform, which appears in the analysis of the eigenvalues of random matrices. However, we are unaware of a formal connection. This potential function is also related to, but is not identical to, the logarithmic barrier function used in Interior Point Algorithms for Linear Programming.
} 
where the barrier $b$ is a real number that varies from step to step.

Initially $A=0$, the barrier is at $b=b_{0}>0$, and the potential is

$$
\Phi_{b_{0}}(0)=\operatorname{Tr}\left[L^{T}\left(0-b_{0} I\right)^{-1} L\right]=-\operatorname{Tr}\left[L^{T} L\right] / b_{0}=-\frac{\|L\|_{F}^{2}}{b_{0}} .
$$

Each step of the process involves adding some rank-one matrix $w w^{T}$ to $A$ where $w \in\left\{L v_{i}\right\}_{i \leq m}$ (if $w=L v_{j}$ then this corresponds to adding $j$ to $\sigma$ ) and shifting the barrier towards zero by some fixed amount $\delta>0$, without increasing the potential. Specifically, we want

$$
\Phi_{b-\delta}\left(A+w w^{T}\right) \leq \Phi_{b}(A) .
$$

We will maintain the invariant that after $k$ vectors have been added, $A$ has exactly $k$ nonzero eigenvalues, all greater than $b$. Keeping the potential small (in fact, sufficiently negative) will ensure that there is a suitable vector to add at each step.

In any step of the process, we are only interested in vectors $w$ which add a new nonzero eigenvalue that is greater than $b^{\prime}=b-\delta$. These are identified in the following lemma, where the notation $A \succeq B$ means that $A-B$ is positive semidefinite.

Lemma 3. Suppose $A \succeq 0$ has $k$ nonzero eigenvalues, all greater than $b^{\prime}>0$. If $w \neq 0$ and

$$
w^{T}\left(A-b^{\prime} I\right)^{-1} w<-1
$$

then $A+w w^{T}$ has $k+1$ nonzero eigenvalues greater than $b^{\prime}$.

Proof. Let $\lambda_{1} \geq \cdots \geq \lambda_{k}$ be the nonzero eigenvalues of $A$, and let $\lambda_{1}^{\prime} \geq \cdots \geq \lambda_{k+1}^{\prime}$ be the $k+1$ largest eigenvalues of $A+w w^{T}$. As the latter matrix is obtained from $A$ by the addition of a rank one positive semi-definite matrix, their eigenvalues interlace [1]:

$$
\lambda_{1}^{\prime} \geq \lambda_{1} \geq \lambda_{2}^{\prime} \geq \cdots \geq \lambda_{k} \geq \lambda_{k+1}^{\prime}
$$

Consider the quantity

$$
\operatorname{Tr}\left[\left(A-b^{\prime} I\right)^{-1}\right]=\sum_{i \leq k} \frac{1}{\lambda_{i}-b^{\prime}}+\sum_{i>k} \frac{1}{0-b^{\prime}},
$$

where we have written the positive and negative terms in the sum separately. By the ShermanMorisson formula,

$$
\operatorname{Tr}\left[\left(A+w w^{T}-b^{\prime} I\right)^{-1}\right]-\operatorname{Tr}\left[\left(A-b^{\prime} I\right)^{-1}\right]=-\frac{w^{T}\left(A-b^{\prime} I\right)^{-2} w}{1+w^{T}\left(A-b^{\prime} I\right)^{-1} w} .
$$

Since $w^{T}\left(A-b^{\prime} I\right)^{-1} w<-1$, the denominator in the right-hand term is negative. The numerator is positive since $A-b^{\prime} I$ is non-singular and $\left(A-b^{\prime} I\right)^{-2} \succeq 0$. So, the right-hand side of (3) is positive.

On the other hand, a direct evaluation of this difference yields

$$
\begin{aligned}
0 & <\operatorname{Tr}\left[\left(A+w w^{T}-b^{\prime} I\right)^{-1}\right]-\operatorname{Tr}\left[\left(A-b^{\prime} I\right)^{-1}\right] \\
& =\frac{1}{\lambda_{k+1}^{\prime}-b^{\prime}}-\frac{1}{0-b^{\prime}}+\sum_{i=1}^{k} \frac{1}{\lambda_{i}^{\prime}-b^{\prime}}-\sum_{i=1}^{k} \frac{1}{\lambda_{i}-b^{\prime}} \\
& \leq \frac{1}{\lambda_{k+1}^{\prime}-b^{\prime}}+\frac{1}{b^{\prime}} \quad \text { since } \frac{1}{\lambda_{i}^{\prime}-b^{\prime}}-\frac{1}{\lambda_{i}-b^{\prime}} \leq 0 \text { for all } i \text { by interlacing. }
\end{aligned}
$$

As $\lambda_{k+1}^{\prime} \geq 0$, this is only possible if $\lambda_{k+1}^{\prime}>b^{\prime}$, as desired. 
The updated potential after one step, as the barrier moves from $b$ to $b^{\prime}=b-\delta$, can be calculated using the Sherman-Morisson formula:

$$
\begin{aligned}
\Phi_{b^{\prime}}\left(A+w w^{T}\right) & =\operatorname{Tr}\left[L^{T}\left(A-b^{\prime} I+w w^{T}\right)^{-1} L\right] \\
& =\operatorname{Tr}\left[L^{T}\left(A-b^{\prime} I\right)^{-1} L\right]-\frac{\operatorname{Tr}\left[L^{T}\left(A-b^{\prime} I\right)^{-1} w w^{T}\left(A-b^{\prime} I\right)^{-1} L\right]}{1+w^{T}\left(A-b^{\prime} I\right)^{-1} w} \\
& =\operatorname{Tr}\left[L^{T}\left(A-b^{\prime} I\right)^{-1} L\right]-\frac{w^{T}\left(A-b^{\prime} I\right)^{-1} L L^{T}\left(A-b^{\prime} I\right)^{-1} w}{1+w^{T}\left(A-b^{\prime} I\right)^{-1} w} \\
& =\Phi_{b^{\prime}}(A)-\frac{w^{T}\left(A-b^{\prime} I\right)^{-1} L L^{T}\left(A-b^{\prime} I\right)^{-1} w}{1+w^{T}\left(A-b^{\prime} I\right)^{-1} w} .
\end{aligned}
$$

To prevent an increase in potential, we want choose a $w$ such that

$$
\Phi_{b^{\prime}}(A)-\frac{w^{T}\left(A-b^{\prime} I\right)^{-1} L L^{T}\left(A-b^{\prime} I\right)^{-1} w}{1+w^{T}\left(A-b^{\prime} I\right)^{-1} w} \leq \Phi_{b}(A) .
$$

We can now determine how small we need the potential to be in order to guarantee that a suitable $w$, which will allow us to keep on going, always exists.

Lemma 4. Suppose $A$ has $k$ nonzero eigenvalues, all of which are greater than $b$, and let $Q$ be the orthogonal projection onto the kernel of $A$. If

$$
\Phi_{b}(A) \leq-m-\frac{\|L\|_{2}^{2}}{\delta}
$$

and

$$
0<\delta<b \leq \delta \frac{\|Q L\|_{F}^{2}}{\|L\|_{2}^{2}}
$$

then there exists a vector $w \in\left\{L v_{i}\right\}_{i \leq m}$ for which $A+w w^{T}$ has $k+1$ nonzero eigenvalues greater than $b^{\prime}=b-\delta$ and $\Phi_{b^{\prime}}\left(A+w w^{T}\right) \leq \Phi_{b}(A)$.

Proof. 2 The vectors satisfying both of the inequalities (2) and (4) are precisely those $w$ for which

$$
\begin{aligned}
& w^{T}\left(A-b^{\prime} I\right)^{-1} L L^{T}\left(A-b^{\prime} I\right)^{-1} w \\
& \quad \leq\left(\Phi_{b}(A)-\Phi_{b^{\prime}}(A)\right) \cdot\left(-1-w^{T}\left(A-b^{\prime} I\right)^{-1} w\right) .
\end{aligned}
$$

We can show that such a $w$ exists by taking the sum over all $w \in\left\{L v_{i}\right\}_{i \leq m}$ and ensuring that the inequality holds in the sum, i.e., that

$$
\begin{aligned}
\operatorname{Tr}[ & \left.L^{T}\left(A-b^{\prime} I\right)^{-1} L L^{T}\left(A-b^{\prime} I\right)^{-1} L\right] \\
& \left.\leq\left(\Phi_{b}(A)-\Phi_{b^{\prime}}(A)\right) \cdot\left(-m-\operatorname{Tr}\left[L^{T}\left(A-b^{\prime} I\right)^{-1} L\right)\right]\right) .
\end{aligned}
$$

Let $\Delta_{b}:=\Phi_{b}(A)-\Phi_{b^{\prime}}(A)$. From the assumption $\Phi_{b}(A) \leq-m-\frac{\|L\|_{2}^{2}}{\delta}$ we immediately have

$$
\operatorname{Tr}\left[L^{T}\left(A-b^{\prime} I\right)^{-1} L\right]=\Phi_{b}(A)-\Delta_{b} \leq-m-\frac{\|L\|_{2}^{2}}{\delta}-\Delta_{b}
$$

\footnotetext{
${ }^{2}$ We would like to thank Pete Casazza for pointing out an important mistake in an earlier version of this proof.
} 
and so (7) will follow from

$$
\operatorname{Tr}\left[L^{T}\left(A-b^{\prime} I\right)^{-1} L L^{T}\left(A-b^{\prime} I\right)^{-1} L\right] \leq \Delta_{b} \cdot\left(\frac{\|L\|_{2}^{2}}{\delta}+\Delta_{b}\right) .
$$

Noting that $L L^{T} \preceq\|L\|_{2}^{2} I$, we can bound the left hand side as

$$
\operatorname{Tr}\left[L^{T}\left(A-b^{\prime} I\right)^{-1} L L^{T}\left(A-b^{\prime} I\right)^{-1} L\right] \leq\|L\|_{2}^{2} \operatorname{Tr}\left[L^{T}\left(A-b^{\prime} I\right)^{-2} L\right] .
$$

Let $P$ be the projection onto the image of $A$ and let $Q$ be the projection onto its kernel, so that $P+Q=I$. Let $\Phi_{b^{\prime}}^{P}(A)=\operatorname{Tr}\left[L^{T} P\left(A-b^{\prime} I\right)^{-1} P L\right]$ and $\Phi_{b^{\prime}}^{Q}(A)=\operatorname{Tr}\left[L^{T} Q\left(A-b^{\prime} I\right)^{-1} Q L\right]$ be the potentials computed on these subspaces. Since $P, Q, A,\left(A-b^{\prime} I\right)^{-1}$, and $\left(A-b^{\prime} I\right)^{-2}$ are mutually diagonalizable, we can write

$$
\begin{gathered}
\Phi_{b^{\prime}}(A)=\Phi_{b^{\prime}}^{P}(A)+\Phi_{b^{\prime}}^{Q}(A), \quad \Delta_{b}=\Delta_{b}^{P}+\Delta_{b}^{Q}, \quad \text { and } \\
\operatorname{Tr}\left[L^{T}\left(A-b^{\prime} I\right)^{-2} L\right]=\operatorname{Tr}\left[L^{T} P\left(A-b^{\prime} I\right)^{-2} P L\right]+\operatorname{Tr}\left[L^{T} Q\left(A-b^{\prime} I\right)^{-2} Q L\right] .
\end{gathered}
$$

As $P\left(A-b^{\prime} I\right)^{-1} P \succeq 0$ and $P(A-b I)^{-1} P \succeq 0$, it is easy to check that

$$
\left(b-b^{\prime}\right) P\left(A-b^{\prime} I\right)^{-2} P \preceq P(A-b I)^{-1} P-P\left(A-b^{\prime} I\right)^{-1} P
$$

which immediately gives

$$
\|L\|_{2}^{2} \operatorname{Tr}\left[L^{T} P\left(A-b^{\prime} I\right)^{-2} P L\right] \leq \Delta_{b}^{P} \frac{\|L\|_{2}^{2}}{\delta} .
$$

Thus, by (8), (9), and (10), we are done if we can show that

$$
\|L\|_{2}^{2} \operatorname{Tr}\left[L^{T} Q\left(A-b^{\prime} I\right)^{-2} Q L\right] \leq\left(\Delta_{b}^{P}+\Delta_{b}^{Q}\right) \cdot\left(\frac{\|L\|_{2}^{2}}{\delta}+\Delta_{b}\right)-\Delta_{b}^{P} \frac{\|L\|_{2}^{2}}{\delta} .
$$

Taking into account that $\Delta_{b}^{P}, \Delta_{b}^{Q} \geq 0$, this is implied by the statement

$$
\|L\|_{2}^{2} \operatorname{Tr}\left[L^{T} Q\left(A-b^{\prime} I\right)^{-2} Q L\right] \leq \Delta_{b}^{Q} \cdot\left(\frac{\|L\|_{2}^{2}}{\delta}+\Delta_{b}^{Q}\right) .
$$

We now compute $\operatorname{Tr}\left[L^{T} Q\left(A-b^{\prime} I\right)^{-2} Q L\right]=\frac{\|Q L\|_{F}^{2}}{b^{\prime 2}}$ and

$$
\left.\Delta_{b}^{Q}=\operatorname{Tr}\left[L^{T} Q\left((A-b I)^{-1}-\left(A-b^{\prime} I\right)^{-1}\right)\right) Q L\right]=\delta \frac{\|Q L\|_{F}^{2}}{b b^{\prime}}
$$

which upon substituting and rearranging reduces (11) to

$$
\|L\|_{2}^{2} \leq \frac{\delta\|Q L\|_{F}^{2}}{b}
$$

which we have assumed in (6) . 
Proof of Theorem 2. We set

$$
b_{0}=\frac{(1-\epsilon)\|L\|_{F}^{2}}{m} \quad \text { and } \quad \delta=\frac{(1-\epsilon)\|L\|_{2}^{2}}{\epsilon m} .
$$

Requirement (5) of Lemma 4 is satisfied at the beginning of the process as

$$
\Phi_{b_{0}}(0)=-\frac{\|L\|_{F}^{2}}{b_{0}}=-m-\frac{\|L\|_{2}^{2}}{\delta} .
$$

To verify that requirement (6) is satisfied initially, first note that the theorem is vacuously true if $\epsilon^{2} \frac{\|L\|_{F}^{2}}{\|L\|_{2}^{2}}<1$. Assuming the converse and recalling that $\epsilon<1$, we may show $\frac{\|L\|_{F}^{2}}{\|L\|_{2}^{2}} \geq 1 / \epsilon$ which implies that $\delta<b_{0}$. The inequality

$$
b_{0} \leq \delta \frac{\|Q L\|_{F}^{2}}{\|L\|_{2}^{2}}
$$

is initially true as $A=0$ and so $Q=\operatorname{Proj}_{\operatorname{ker}(A)}=I$.

As long as condition (6) is satisfied, we may apply Lemma 4 to add a vector to $\sigma$ while maintaining $\Phi_{b}(A) \leq \Phi_{b_{0}}(0)$. The left-hand inequality in (6) will be satisfied after the first $t-1$ steps if

$$
\delta<b=b_{0}-(t-1) \delta \quad \Longleftrightarrow \quad t \delta<b_{0} .
$$

This inequality is satisfied for all $t \leq \epsilon^{2} \frac{\|L\|_{F}^{2}}{\|L\|_{2}^{2}}$ as

$$
\epsilon^{2} \frac{\|L\|_{F}^{2}}{\|L\|_{2}^{2}} \delta=\frac{\epsilon(1-\epsilon)\|L\|_{F}^{2}}{m}<b_{0} .
$$

The right-hand inequality in (6) will always be satisfied if it is satisfied initially as the Frobenius norm $\|Q L\|_{F}^{2}$ decreases by at most $\|L\|_{2}^{2}$ in each step. Taking $t=\left\lfloor\epsilon^{2} \frac{\|L\|_{F}^{2}}{\|L\|_{2}^{2}}\right\rfloor$ steps leaves the barrier at

$$
b_{0}-\delta t \geq \frac{(1-\epsilon)\|L\|_{F}^{2}}{m}-\epsilon^{2}(1-\epsilon) \frac{\|L\|_{F}^{2}}{\epsilon m}=\frac{(1-\epsilon)^{2}\|L\|_{F}^{2}}{m}
$$

which is the promised bound.

\section{Acknowledgements}

We would like to thank Kate Juschenko, Roman Vershynin and especially Pete Casazza for helpful comments and corrections to an earlier version of this manuscript.

\section{References}

[1] Joshua D. Batson, Daniel A. Spielman, and Nikhil Srivastava. Twice-Ramanujan sparsifiers. In STOC '09: Proceedings of the 41st annual ACM symposium on Theory of computing, pages 255-262, New York, NY, USA, 2009. ACM.

[2] K. Berman, H. Halpern, V. Kaftal, and G. Weiss. Matrix norm inequalities and the relative Dixmier property. Integral Equations and Operator Theory, 11:28-48, 1988. 
[3] J. Bourgain and L. Tzafriri. Invertibility of large submatrices with applications to the geometry of Banach spaces and harmonic analysis. Israel Journal of Mathematics, 57:137$224,1987$.

[4] J. Bourgain and L. Tzafriri. On a problem of Kadison and Singer. J. Reine Angew. Math., 420:1-43, 1991.

[5] P. Casazza and J. Tremain. Revisiting the Bourgain-Tzafriri Restricted Invertibility Theorem. Operators and Matrices, 3:97-110, 2009.

[6] Joel A. Tropp. Column subset selection, matrix factorization, and eigenvalue optimization. In SODA '09: Proceedings of the Nineteenth Annual ACM -SIAM Symposium on Discrete Algorithms, pages 978-986, Philadelphia, PA, USA, 2009. Society for Industrial and Applied Mathematics.

[7] R. Vershynin. John's decompositions: Selecting a large part. Israel Journal of Mathematics, 122:253-277, 2001. 